ВЕСТНИК НПУА. МЕТАЛЛУРГИЯ, МАТЕРИАЛОВЕДЕНИЕ, НЕДРОПОЛЬЗОВАНИЕ. 2021. №1 УДК 537.531

DOI: 10.53297/18293395-2021.1-63

\title{
ИССЛЕДОВАНИЕ ПОЛЕЙ ДЕФОРМАЦИЙ В ЗЕРКАЛЬНОМ КРИСТАЛЛЕ РЕНТГЕНОВСКОГО ИНТЕРФЕРОМЕТРА, ПОДВЕРГНУТОГО ИОННОЙ ИМПЛАНТАЦИИ
}

\author{
А.О. Абоян
}

Нацииональный политехнический университет Армении

Обзор научной литературы по теории интерференции рентгеновских лучей показывает, что вопросам изучения тонких имплантированных слоев рентгенодифракционными методами посвящено много работ. Все эти работы имеют некоторые общие характерные черты. В частности, все рентгенотопографические исследования проводились с образцами, которые облучались энергиями выше 1 МэB.

При облучении ионами с низкими энергиями, в особенности в малых дозах, необходимо пользоваться рентгеноинтерферометрическими методами, чувствительность и разрешение которых несравнимо велики. В настоящей работе приведены результаты теоретических и экспериментальных рентгеноинтерферометрических исследований несовершенств кристаллов, возникающих в результате ионной имплантации малыми дозами и низкими энергиями.

Результаты исследований представлены с помощью рентгенодифракционных муаровых картин полей деформаций, возникающих в одной из работающих частей зеркального блока трехкристального рентгеновского кремниевого интерферометра, подвергнутого ионной имплантации, в зависимости от дозы облучения. Исследовано также перераспределение напряжений, возникающих в этом участке блока интерферометра при его бомбардировке. Полученные результаты дают основу для решения обратной задачи, а именно - задачи восстановления полей механических напряжений в кристаллических блоках интерферометра путем расшифровки муаровых картин, так как изменение муаровых картин будет заключать в себе информацию о механических напряжениях. Таким образом, метод рентгеноинтерферометрического муара дает возможность прямого экспериментального изучения полей механических напряжений, созданных структурными дефектами кристаллической решетки. Выведено условие применимости выражения для определения периода муаровых картин при оценке внутрикристальных деформаций.

Ключевые слова: монокристалл кремния, трехкристальный интерферометр, муаровые картины, ионная имплантация, доза облучения, поле деформаций, относительная деформация.

Введение. Создание различного типа рентгеновских интерферометров и расшифровка полученных интерференционных (муаровых) картин позволили решить ряд актуальных задач, в частности, исследовать структурные дефекты 
в монокристаллах. Преимущество интерферометрического метода заключается в том, что на рентгеновской муаровой картине отображаются не только структурные дефекты, но и их поля напряжений вдали от дефектов.

Однако во всех интерферометрических исследованиях важными условиями являются расшифровка муаровых картин (оценка внутрикристальных деформаций исходя из вида муара), а также подбор и упрощение выражения для вычисления периодов муаровых картин.

Известно [1,2], что при бомбардировке монокристаллов ионами больших энергий ( 80 кэ $B \ldots 3$ Мэ $B$ ) в них возникают радиационные нарушения, сводящиеся, в частности, к изменениям межплоскостных расстояний, и, следовательно, в кристалле возникает напряженное состояние.

В работах $[3,4]$ показано, что в случае ионной имплантации одного из блоков рентгеновского интерферометра возникают поперечные деформации. В связи с этим интерес представляет исследование влияния внешнего воздействия (бомбардировка ионами) на степень возникающих при этом структурных несовершенств путем изменения периода дилатационных муаровых картин, получаемых при использовании рентгеновского интерферометра, состоящего из трех кристаллических пластинок.

В настоящей работе приводятся экспериментальные и теоретические результаты исследования методом рентгенодифракционного муара полей деформаций, возникающих в одной из работающих частей блоков кремниевого рентгеновского интерферометра, подвергнутого ионной имплантации, в зависимости от дозы облучения. Исследовано также перераспределение напряжений, возникающих в этом участке блока интерферометра при его бомбардировке. Выведено условие применения известного выражения [5] для вычисления периодов муаровых картин с целью оценки внутрикристальных деформаций.

Теоретический анализ. Известно, что относительные деформации (дилатации) кристаллической решетки можно вычислить с помощью выражения

$$
\frac{\Delta d}{d}=\frac{d}{\Lambda_{\|}}
$$

где $d$-период решетки; $\Lambda_{\|}-$период параллельного (дилатационного) муара.

В работе [6] показано, что для изучения поперечных напряжений, вызванных ионной бомбардировкой кристаллов кремния, выражение (1) неприменимо, так как оно верно только для двухкристальной системы, т.е. когда рентгеновская волна последовательно дифрагирует в двух кристаллах, 
различающихся периодами. Но природа возникновения муаровой картины от рентгеновского интерферометра иная, так как в интерферометре облучаются не две области, а четыре (рис. 1), и у входной поверхности последнего блока (блока-анализатора) происходит наложение когерентных волн (явление интерференции).

Следовательно, используя формулу (1) для вычисления относительных деформаций решетки с помощью муаровых картин, возникающих от рентгеновского интерферометра, получаем их усредненные значения. Это значит, что выражение (1) можно использовать для вычисления периодов интерферометрического муара, если из четырех облучаемых областей три области идентичны.

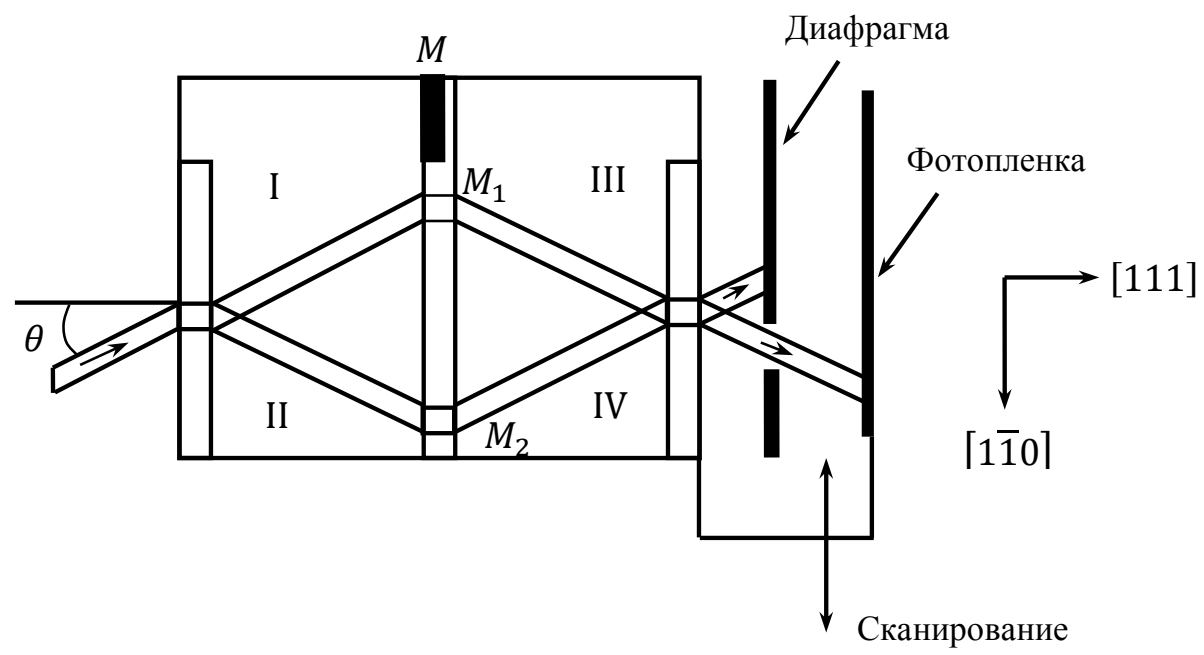

Рис. 1. Ход лучей в трехкристальном интерферометре

Предположим, что три облученные области имеют один и тот же период решетки $d_{0}$, а четвертый - период $d$ (рис. 1). Тогда возникает дилатационный муар с периодом

$$
\Lambda_{\|}=\frac{d_{0} d}{\left|d_{0}-d\right|}=\frac{d_{0} d}{\Delta d} \text { или } \frac{\Delta d}{|d|}=\frac{d_{0}}{\Lambda_{\|}} .
$$

В работе [7] при имплантации ионов в кристаллической пластинке для интегрального напряжения получено следующее выражение:

$$
\sigma=\alpha \frac{E D}{1-v} \quad \text { или } \quad \frac{\sigma}{E}=\alpha \frac{D}{1-v},
$$


где $D$-доза облучения, выраженная в количестве ионов на единицу площади; $E$ - модуль Юнга; $v$ - коэффициент Пуассона; $\sigma$ - внутреннее напряжение; $\alpha$ - решеточный коэффициент расширения. Так как ось $Z$ в нашем случае параллельна направлению [111], а ось $X$ - направлению [11̄0] (рис. 2), то согласно [8], $E=E_{x}=1,689 \cdot 10^{11} H / M^{2}, v=v_{x}=0,262$.

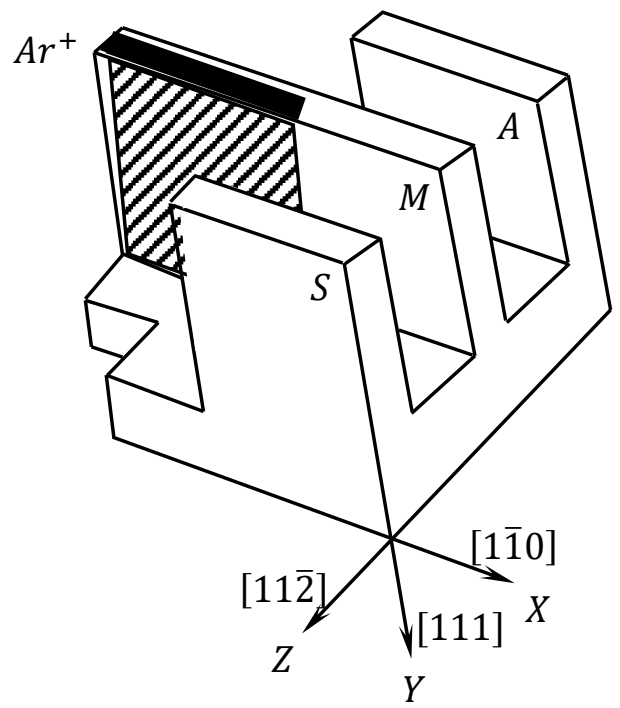

Рис. 2. Трехкристальный интерферометр с выбранной кристаллографической ориентацией

Имея в виду, что относительная деформация кристаллической решетки приводит к изменению периода дилатационного муара, с учетом (2) получим

$$
\frac{\Delta d}{d}=\frac{\sigma}{E}=\frac{d_{0}}{\Lambda_{\|}}
$$

где $\Delta d-$ абсолютное изменение периода отражающих плоскостей.

Из (3) и (4) получим $\frac{d_{0}}{\Lambda_{\|}}=\alpha \frac{D}{1-v}$, откуда имеем

$$
\Lambda_{\|}=\frac{d_{0}(1-v)}{\alpha D} \text {. }
$$

Как видно из (5), период дилатационной муаровой картины обратно пропорционален дозе облучения.

Экспериментальная часть. Для подтверждения справедливости вышеуказанных теоретических рассуждений проведены экспериментальные исследования с использованием высокосовершенного монокристального интерферометра (рис. 2), зеркальный блок которого на 9 мм шире, чем остальные 
два блока, что позволяет получить информацию только из этой области зеркального блока интерферометра (заштрихованная часть на рис. 2). Толщина блоков составила 0,8 мм , ширина - 22 мм (ширина зеркального блока - 31 мм), высота - 18 мм, межблочное расстояние - 14 мм (использовано $\mathrm{Cu} \mathrm{K}_{\alpha}$ - излучение).

Для определения напряжений и относительных деформаций, вызванных ионным легированием, вначале (до имплантации) сканированием интерферометра (рис. 1) снималась муаровая топограмма (рис. 3а) от интерферометра для отражения $2 \overline{2} 0$.

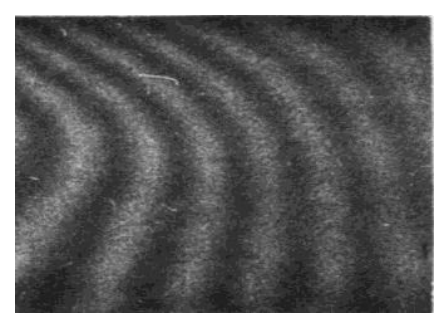

a)

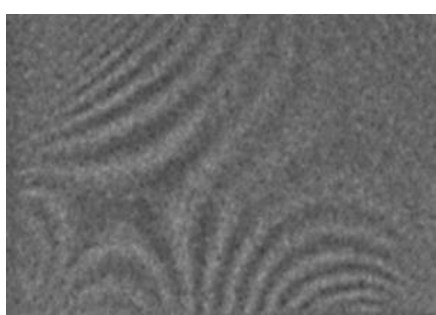

б)

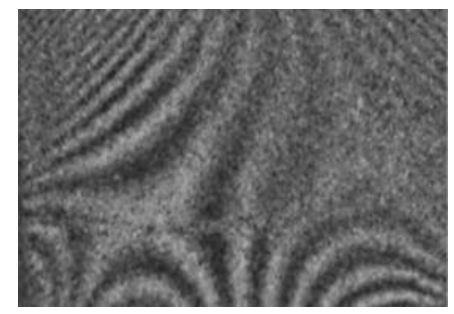

д)

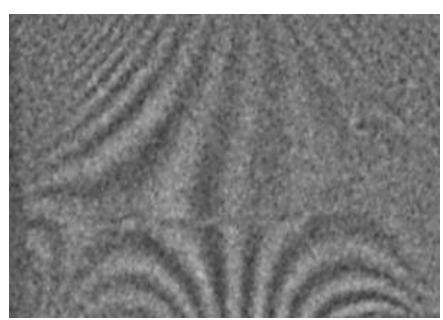

B)

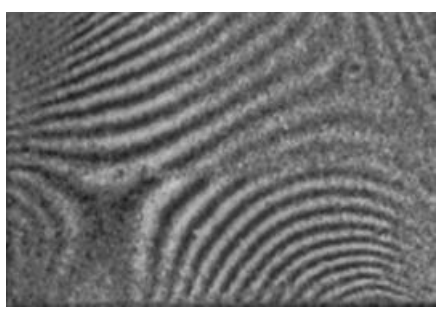

e)

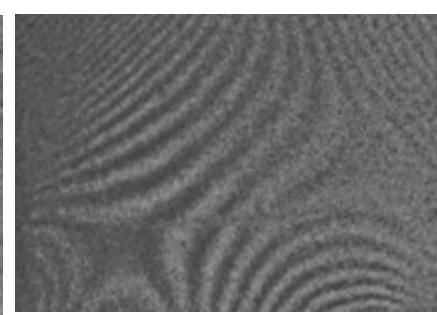

г)

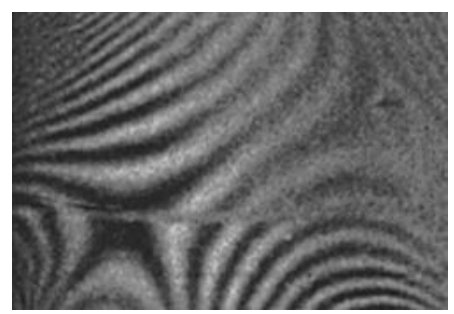

ж)

Рис. 3. Исходная муаровая топограмма до генерирования дислокаций и до имплантации (а). Муаровая топограмма после генерирования $60^{0}$ дислокаций (б). Топограммы после ионной имплантаџии (в-ж). Доза облучения: $10^{13} \mathrm{uон/ \textrm {cm } ^ { 2 }}$ (в), $10^{14} \mathrm{uон} / \mathrm{cm}^{2}$ (2), $10^{15}$ ион/ $\mathrm{cm}^{2}\left(\right.$ ()), $10^{16} \mathrm{ион} / \mathrm{cm}^{2}\left(\right.$ e), $10^{17}$ ион/ $\mathrm{cm}^{2}$ (ж) 
Как видно из исходной муаровой топограммы (рис. 3a), монокристалл $S i$, из которого изготовлен интерферометр, достаточно совершенный, так как на топограмме получено всего шесть муаровых линий (периоды муаровых полос очень большие). Как известно, если интерферометр изготовлен из абсолютно идеального кристалла, т.е. из монокристалла без внутренних напряжений, то муаровые узоры не получаются.

Далее в избыточной области $M_{1}$ (рис. 1) генерировано $60^{\circ}$ дислокаций методом, описанным в [9], и снята муаровая топограмма (рис. 3б). Из сравнения этой топограммы с топограммой, показанной на рис. За, становится очевидным, что формирование муаровой картины обусловлено внесением дислокаций, т.е. пучком III (рис. 1), который отражается вблизи области скопления дислокаций.

После получения исходной топограммы (рис. 3б) одна из поверхностей избыточной части зеркального блока интерферометра была подвергнута имплантации ионами аргона с энергией $180 \kappa э B$. Суммарная доза менялась в пределах от $10^{13}$ до $10^{17} \mathrm{uoн} / \mathrm{cm}^{2}$. После каждого акта облучения ионами аргона проводились съемки, в результате чего были получены топограммы, соответствующие различным дозам облучения (рис. Зв-ж). Во всех случаях облучаются одни и те же участки интерферометра.

Обсуждение результатов. Исходя из полученных с помощью топограмм микрометрических кривых, в зависимости от дозы облучения, на основе выражений (4) и (5) вычислены относительные деформации при имплантации в кремний ионов аргона с энергией 180 кэ $B$. Результаты приведены в таблице.

Таблица

Относительные деформации в зависимости от дозы облучения

\begin{tabular}{|c|c|c|}
\hline № & 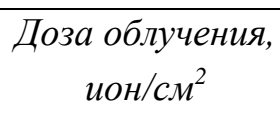 & $\begin{array}{c}\text { Относительные деформации, } \\
\qquad \varepsilon=|\Delta d / d|\end{array}$ \\
\hline 1 & $10^{13}$ & $2.21 \cdot 10^{-7}$ \\
\hline 2 & $10^{14}$ & $2.39 \cdot 10^{-7}$ \\
\hline 3 & $10^{15}$ & $2.58 \cdot 10^{-7}$ \\
\hline 4 & $10^{16}$ & $2.68 \cdot 10^{-7}$ \\
\hline 5 & $10^{17}$ & $2.37 \cdot 10^{-7}$ \\
\hline
\end{tabular}

Как видно из табличных данных, зависимость относительной

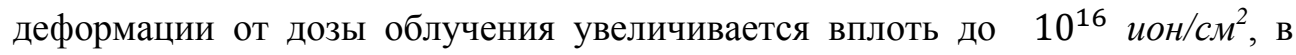
отличие от результатов работы [4], где относительная деформация достигает 


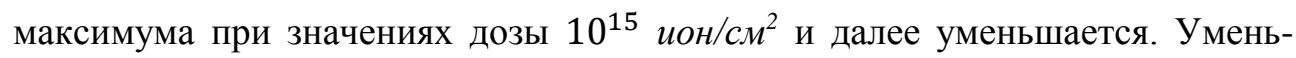
шение объясняется тем, что аморфизация слоя, подвергнутого имплантации, приводит к изменению коэффициента расширения решетки, величина которого перестает быть константной. Как видно из топограмм, приведенных на рис. 3в-ж, облучение ионами приводит к изменению муаровых полос.

С увеличением дозы облучения период муаровых полос сначала уменьшается (деформация увеличивается) (рис. Зв-е), а затем увеличивается (деформация уменьшается) (рис. Зж). Из этих рисунков видно, что при имплантации возникают дислокации.

Наблюдаемые на топограммах дилатационные муаровые картины дают возможность отделить напряжения, внесенные при имплантации, от имевших место в исходном образце, а также показать динамику изменения муаровых картин в зависимости от дозы облучения. Сопоставление периодов муаровых картин для различных доз облучения показывает, что по сравнению с областью $M_{1}$, как область $M_{2}$, так и области отражений пучков в блоках $S$ и $A$ (рис. 1) можно считать совершенными.

Заключение. Полученные результаты дают основу для решения обратной задачи, а именно, задачи восстановления полей механических напряжений в кристаллических блоках интерферометра путем расшифровки муаровых картин, так как изменение муаровых картин будет заключать в себе информацию о механических напряжениях.

Таким образом, метод рентгеноинтерферометрического муара дает возможность прямого экспериментального изучения полей механических напряжений, созданных структурными дефектами кристаллической решетки. Область муара, непрерывно меняющаяся с изменением дозы облучения, может быть использована для вычисления изменения механического напряжения в кристаллическом блоке интерферометра. При расшифровке рентгеноинтерферометрических муаровых картин можно использовать выражение (1) только в том случае, если в одной из отражающих областей любого из трех блоков имеются значительные нарушения периодичности атомных плоскостей по сравнению с остальными тремя облучаемыми областями интерферометра.

\section{Литература}

1. Bonse U., Hart M., Schuttke G.H. // Phys. Status. Solidi.- 1969. - V.33.- P. 361.

2. Schuttke G.H., Brack K. // Z. Naturforsh. - 1973.- V.281. - P.654.

3. Gerward L. // Z. Physik. - 1973. - V. 259.- P.313.

4. Абоян А.О. // Известия НАН Армении. Физика. - 2000. - Т.35, №3. - С. 212.

5. Электронная микроскопия тонких кристаллов /П. Хирш, А. Хови, Р. Николсон и др.- М.: Мир, 1968. - 574 с. 
6. Дрмеян Г. Р. // Кристаллография. -2005. - Т. 50, № 3.- С.407.

7. Дрмеян Г. Р., Абоян А.О. // Поверхность. Рентген., синхротр. и нейтрон. исслед. - 2006.- №12.- С. 47 -52.

8. Wortman I. J., Ewans R.A. // Appl. Phys. -1965. - V. 36. - P. 153.

9. Багдасарян Р.И., Мнацаканян Т.О., Эйрамджян Ф.О. и др. // Уч. зап. ЕГУ.1983. - T.2. - C. 162.

Поступила в редакиию 25.12.2020.

Принята к опубликованию 21.05.2021 .

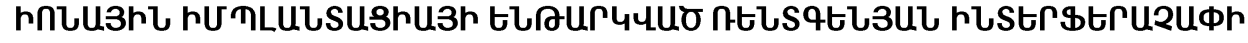

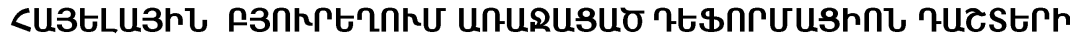 <ESURกSחRA3กRlC}

\section{U.<. Upnjwir}

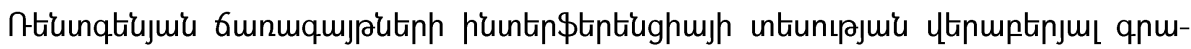

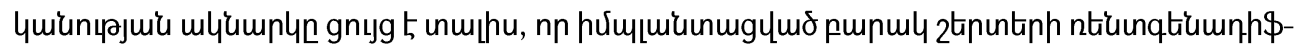

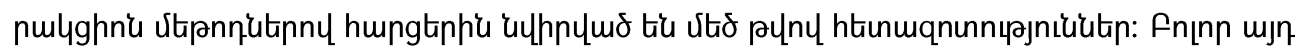

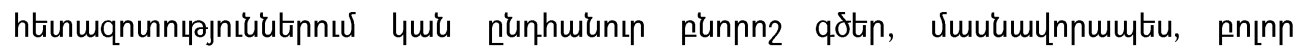

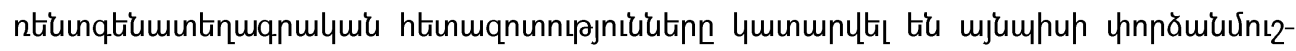

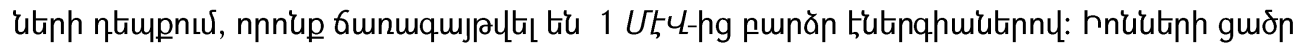

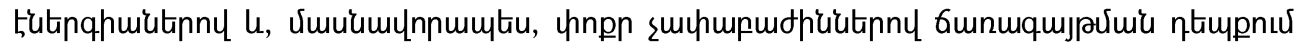

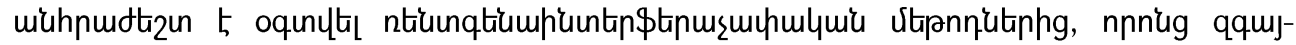

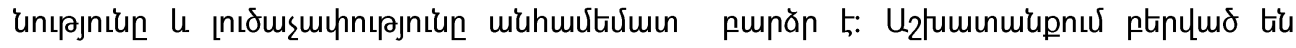

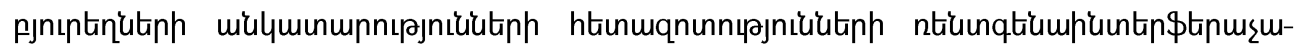

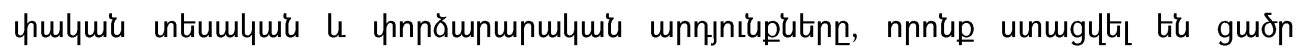

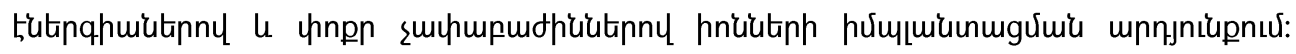

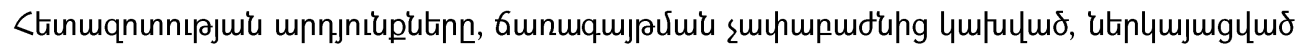

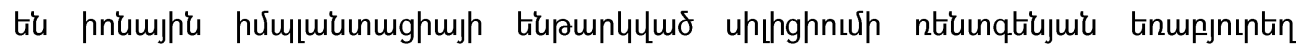

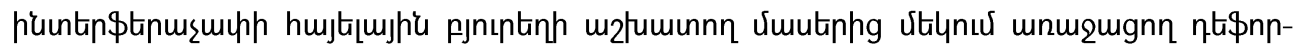

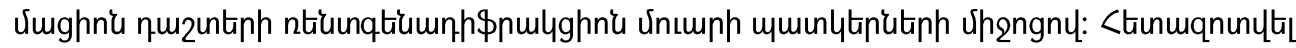

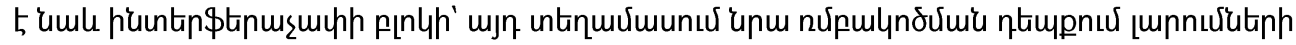

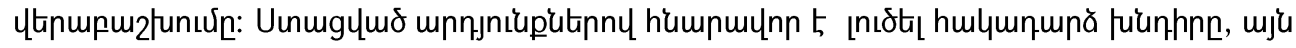

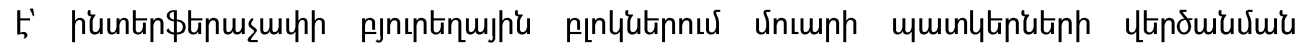

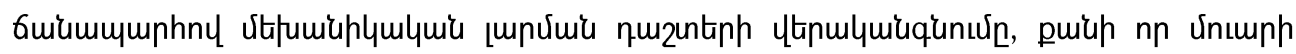

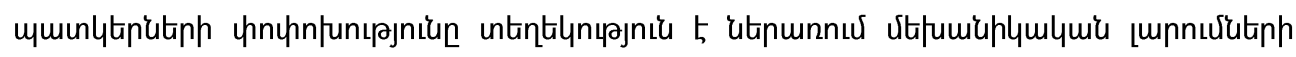

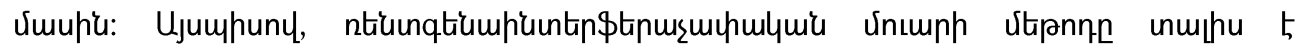

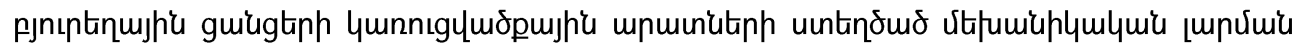

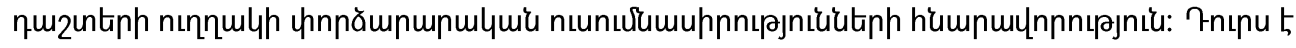




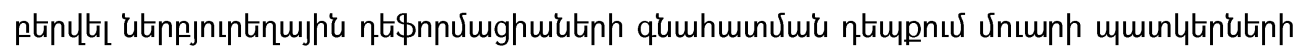

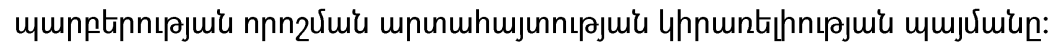

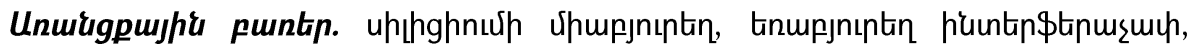

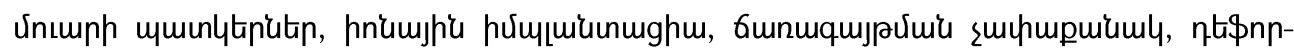

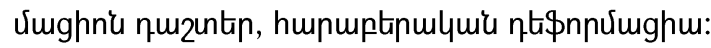

\title{
INVESTIGATING THE DEFORMATION FIELDS IN A MIRROR CRYSTAL OF AN X- RAY INTERFEROMETER UNDERGONE IONIC IMPLANTATION
}

\begin{abstract}
A.H. Aboyan
The review of scientific literature on the theory of the X-ray interference shows that many investigations have been devoted to the issues on the thin implanted layers by X-ray diffraction methods. All these studies have certain common characteristic features. In particular, all X-ray topographic investigations have been carried out with samples radiated by powers higher than $\mathrm{MeV}$.

At radiating with ions of low powers, especially in small doses, it is necessary to apply $\mathrm{X}$-ray interferometric methods whose sensitivity and resolution are incomparably large. In the present work, the results of theoretical and experimental X-ray interferometric investigations of the crystal defects, arising as a result of ionic implantation by small doses and low powers are introduced.

The investigation results are presented by X-ray diffraction moiré patterns of the deformation fields, arising in one of the operating parts of the mirror block of a three -crystal $\mathrm{X}$-ray silicon interferometer subjected to ionic implantation, depending on the radiation dose. The stress redistribution, occurring in that section of the interferometer block at its bombardment is also studied. The results obtained are a basis for solving the inverse problem, namely, the problem of restoring the fields of mechanical stresses in the crystal blocks of the interferometer by interpreting the moiré patterns, as the change in the moiré patterns will include information on mechanical stresses. Thus, the method of the X-ray interferometric moiré allows to carry out a direct experimental investigation of the fields of mechanical stresses created by structural defects of the crystal lattice. The condition for the applicability of the expression for determining the period of the moire patterns at estimating the intracrystal deformations is obtained.
\end{abstract}

Keywords: silicon mono crystal, three-crystal interferometer, moiré patterns, ionic implantation, radiation dose, deformation fields, relative deformation. 